\title{
Efecto de la deficiencia de N, P, K, Mg, Ca y B sobre la acumulación y distribución de la masa seca en plantas de guayaba (Psidium guajava L.) var. ICA Palmira II en fase de vivero
}

\section{Effect of N, P, K, Mg, Ca and B deficiencies on the accumulation and distribution of dry mass in guava plants (Psidium guajava L.) var. Palmira ICA II in the nursery phase}

SINDY LORENA DUSSÁN ${ }^{1}$

DANIEL ANDRÉS VILLEGAS ${ }^{1}$

DIEGO MIRANDA ${ }^{1,2}$

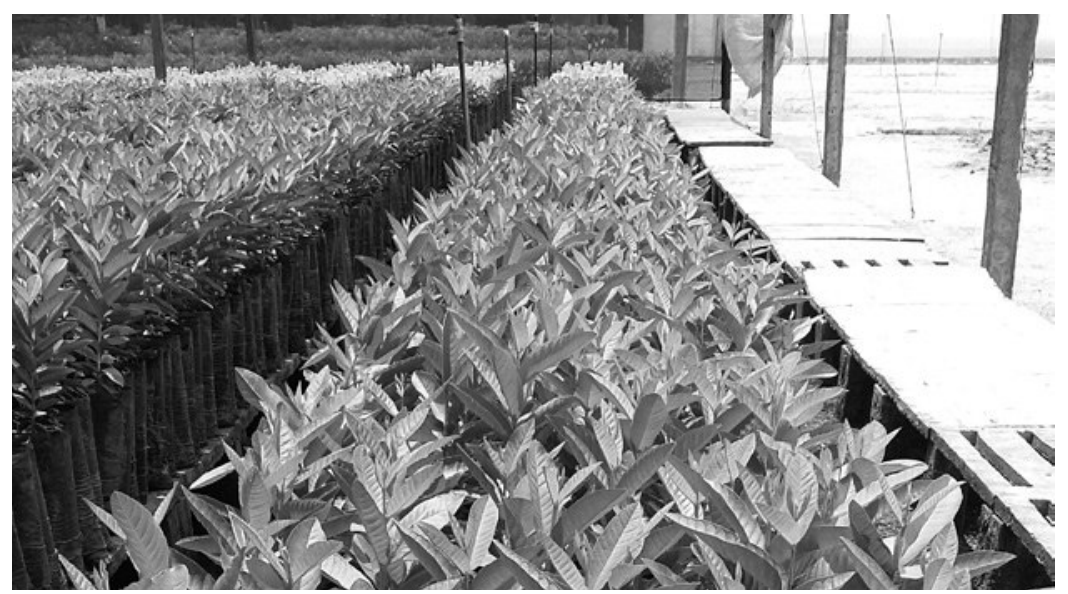

Plantas de guayaba en vivero.

Foto: D. Miranda

\section{RESUMEN}

La guayaba, nativa de América tropical, se destaca por ser una fruta de alto contenido vitamínico. En Colombia, es un cultivo con poca investigación especialmente en el área de la nutrición y fertilización. Bajo invernadero se cultivaron plantas jóvenes de guayaba de tres meses de edad, de la variedad ICA Palmira II, las cuales fueron sometidas a tratamientos con fertilizantes mediante la técnica del elemento faltante, con los siguientes tratamientos: T0: agua; T1: fertilización completa; T2: completa -N; T3: -P; T4: -K; T5: -Mg; T6: -Ca y T7: -B. Las plantas fueron sembradas en sustrato de arena lavada de río y gravilla en bolsas de polietileno negras de (5 L de capacidad); la aplicación de los tratamientos se realizó manualmente con riegos diarios de las soluciones nutritivas diarias con un volumen de $250 \mathrm{~cm}^{3}$ por bolsa. Se determinaron los cambios de magnitud de las plantas: altura y número de hojas, área foliar y la acumulación de masa seca por las estructuras de la planta. Se encontró que el tratamiento -N, en comparación con la fertilización completa, afectó el número de hojas y la acumulación de masa seca por las estructuras de la planta (raíz, tallo, hojas y total). El N resultó ser el elemento más limitante en la fase vegetativa de plantas jóvenes de guayaba (primeros 8 meses del desarrollo). La deficiencia de fósforo (-P) causó reducciones en el área foliar de las plantas (-20\%) en comparación con el tratamiento de fertilización completa; el tratamiento -K redujo el área foliar solo en el 8\%, y -B en 5,4\%. La deficiencia de calcio (-Ca) ocasionó la menor acumulación de masa seca por la raíz, respuesta que estuvo asociada a la relación de este nutriente con la actividad auxínica en las raíces.

\footnotetext{
Facultad de Ciencias Agrarias, Departamento de Agronomía, Universidad Nacional de Colombia, Bogotá (Colombia). Autor para correspondencia.dmirandal@unal.edu.co
} 
Palabras clave adicionales: masa seca, número de hojas, elemento faltante, área foliar.

\section{ABSTRACT}

Guava is native to the tropical Americas and stands out as a fruit with a high vitamin content; however, it is a crop with little research or technical assistance, especially in terms of nutrition and fertilization. In greenhouse, three-month-old guava plants, variety ICA Palmira II, were subjected to fertilizer treatments using the missing element technique. The following treatments were used: T0: water; T1: complete fertilization; T2: complete -N; T3: -P; T4: -K; T5: -Mg; T6: -Ca and T7: -B. The plants were planted in soil with washed river sand and gravel in black polyethylene bags ( $5 \mathrm{~L}$ capacity); the application of the treatments was done manually, with daily watering with the nutrient solutions at a volume of $250 \mathrm{~cm}^{3}$ per bag. The changes in the height, number of leaves, leaf area and dry matter accumulation by the plant structures were determined. We found that the $-\mathrm{N}$ treatment, as compared to the complete fertilization, affected the number of leaves and dry matter accumulation by the structures of the plant (root, stem, leaves and total). $N$ was the most limiting element in the vegetative phase of the young guava plants (first 8 months of development). The deficiency in phosphorus (-P) caused reductions in the plant leaf area $(-20 \%)$, as compared to the complete fertilization treatment; the $(-\mathrm{K})$ treatment reduced the leaf area only by $8 \%$; a $5.4 \%$ reduction was seen in (-B). The calcium deficiency (-Ca ) caused the lowest accumulation of root dry mass; this response was associated with the relationship of this nutrient with the auxin activity in the roots.

Additional keywords: dry mass, leaf number, missing element, leaf area.

Fecha de recepción: 14-12-2016

Aprobado para publicación: 14-05-2016

INTRODUCCIÓN

La guayaba (Psidium guajava L.), especie nativa de América tropical continental, pertenece a la familia Myrtaceae (IIFT, 2011). Las cualidades nutricionales de la fruta hacen que sea considerada como la manzana del trópico (Prakash et al., 2002), siendo la única fruta que contiene hasta 16 vitaminas, ocupando el tercer lugar en su contenido de Vitamina C (MADR, 2007; Fischer et al., 2012). Los principales productores son Brasil, Colombia, Perú, Ecuador, India, Sudáfrica, Estados Unidos, México, Filipinas, Venezuela, Costa Rica, Cuba y Puerto Rico (MINAG, 2011). Para el año 2013, en Colombia el área sembrada correspondió a 23.900 ha y el rendimiento promedio fue de 11,5 t ha-1 (FAO, 2013). Se cultiva en los departamentos de Santander (4.310 ha), Boyacá
(1.849 ha), Valle del Cauca (1.180 ha), Tolima (2.439 ha), Cundinamarca (460 ha), Antioquia (990 ha), Huila (107 ha), Meta (333 ha), Atlántico (338 ha) y Bolívar (238 ha) (MADR, 2007).

Las plantas en general requieren elementos esenciales que les permitan completar su ciclo vegetal de forma óptima, como lo son el $\mathrm{C}, \mathrm{H}$ y $\mathrm{O}$ que provienen del aire y el agua, y a su vez conforman el $95 \%$ de la masa seca de los tejidos vegetales; además, requieren minerales esenciales los cuales deben ser proporcionados de forma exógena de acuerdo con el balance requerimiento - suministro, presentando demanda de macronutrientes ( $\mathrm{N}, \mathrm{P}, \mathrm{K}, \mathrm{Mg}, \mathrm{Ca}$ y $\mathrm{S}$ ) y micronutrientes ( $\mathrm{Cl}, \mathrm{Fe}, \mathrm{B}, \mathrm{Mn}, \mathrm{Zn}, \mathrm{Mo}$ ) y otros 
elementos benéficos (Se, Co, Na, Ni, Si) para ciertos grupos de plantas (Malavolta, 2006). El suministro de nutrientes se realiza teniendo como base los niveles de nutrientes disponibles en el suelo y el estado nutricional de las plantas (Marschner, 2012).

El análisis de nutrientes en plantas es una técnica que determina el contenido de los nutrientes en tejidos vegetales de un cultivo muestreado en un momento o etapa de desarrollo determinados (Munson y Nelson, 1986; Campbell, 2000) y se refiere al análisis cuantitativo de laboratorio de los tejidos vegetales recolectados, siendo el más frecuente el análisis de tejido foliar. Otra técnica utilizada cuando no es factible el análisis de suelos o el tejido foliar es la técnica del elemento faltante, que se hace cultivando plantas utilizando contenedores pequeños en los cuales cada uno de los nutrientes evaluados se omite en un tratamiento, pero todos los demás nutrientes se aplican en niveles adecuados (Bruulsema et al., 2012).

La producción de masa seca (MS) es el resultado del proceso de fotosíntesis; desde el punto de vista cuantitativo esta acumulación y el crecimiento dependen del exceso de carbohidratos sintetizados en relación con aquellos que son usados como sustratos de la respiración. Sin embargo, en el caso particular de las estructuras de la planta este concepto no es necesariamente estricto, ya que el mecanismo de redistribución de los fotosintatos puede incrementar o reducir la biomasa acumulada en cada una de las estructuras de la planta (Norero and Pilatti, 2002). Aproximadamente el $90 \%$ de la masa seca acumulada es producto de la actividad fotosintética, mientras que el porcentaje restante proviene de la absorción mineral (Benincasa, 2003). Cuando los nutrientes minerales son limitantes las plantas reducen su crecimiento y alteran aspectos de su morfología, como también la adquisición y el uso de estos minerales con el fin de maximizar la adquisición de estos recursos escasos (Schachtman y Shin, 2007). De igual manera, la visualización de los síntomas es una herramienta invaluable para la caracterización morfológica (Lizarazo et al., 2013a),

No se tienen estudios para Colombia sobre el efecto de los nutrientes en la fase vegetativa correspondiente al crecimiento en vivero. La fase vegetativa de la guayaba en fase inicial incluye el trasplante hasta los 12 meses de edad de las plantas y se caracteriza por la formación de estructuras vegetativas (tronco principal y las ramas primarias hasta formar la altura de la copa). Por lo anterior, el objetivo de esta investigación fue evaluar el efecto de la deficiencia de N, P, K, Mg, Ca y B mediante la técnica del elemento faltante sobre la acumulación y distribución de la masa seca de plantas jóvenes de guayaba (Psidium guajava L.) variedad ICA Palmira II cultivadas en un sustrato inerte y durante la fase de vivero.

\section{MATERIALES Y MÉTODOS}

\section{Ubicación y material vegetal}

Esta investigación se realizó en un invernadero de plástico de la Facultad de Ciencias Agrarias, Universidad Nacional de Colombia, Bogotá, a una altitud de $2.556 \mathrm{msnm}$ con temperatura y humedad promedio interior de $24^{\circ} \mathrm{C}$ y $65 \%$, respectivamente. Se emplearon 152 árboles de Guayaba var. ICA Palmira II de tres meses de edad propagados a partir de semilla y dispuestas en sustrato de cascarilla de arroz quemada y suelo (relación 1:1), luego fueron trasplantados a bolsas de polietileno con capacidad de $3 \mathrm{~kg}$; el sustrato consistió de una mezcla compuesta con arena de sílice y gravilla con una relación $2: 1$, con un tamaño de grano de 0,06 a 0,1 y $0,5 \mathrm{~mm}$, respectivamente, el cual fue lavado con agua destilada con el fin de remover los minerales que pudieran estar disponibles posteriormente.

Se utilizó un diseño experimental completamente al azar con ocho tratamientos, tres árboles por unidad experimental, con tres repeticiones 
y para seis muestreos, para un total de 432 unidades experimentales. Los tratamientos evaluados fueron: T0: aplicación de agua sin adición de elementos minerales; T1: fertilización completa (testigo absoluto); T2: fertilización completa menos nitrógeno (-N); T3: fertilización completa menos fósforo (-P); T4: fertilización completa menos potasio (-K); T5: fertilización completa menos magnesio (-Mg); T6: fertilización completa menos calcio (-Ca) y T7: fertilización completa menos boro (-B). Las plantas fueron distribuidas en cuatro camas de $1,20 \mathrm{~m}$ separadas entre sí y distanciadas de 0,20 m entre plantas, puestas en hileras dobles.

Los tratamientos de fertilizantes fueron balanceados a partir de la solución Hoagland y Arnon (1950), empleando la técnica del elemento faltante para evaluar los requerimientos nutricionales propuestos por Natale et al. (1996), para los macronutrientes y Salvador et al. (2003) para los micronutrientes. Luego del trasplante de los árboles a las bolsas se les aplicó agua destilada durante dos semanas, pasado este tiempo se inició la aplicación de los tratamientos. Las soluciones concentradas fueron suministradas por Brenntag Colombia S.A. (Bogotá), las cuales se indican en la tabla 1. Cada solución se aplicó con una probeta graduada en una dosis de $500 \mathrm{~mL} \mathrm{día}{ }^{-1}$ por bolsa de forma manual.

Como fuentes comunes se usaron $\mathrm{Mn} 1,8 ; \mathrm{Zn}$ 0,2; Cu 0,08; Fe 0,5 y Mo 0,02 $\mathrm{mg} \mathrm{L}^{-1}$ para todos los tratamientos. Las fuentes utilizadas en la preparación de las soluciones madre fueron: nitrato de amonio líquido, fosfato monopotásico, DAP, nitrato de potasio, sulfato de potasio, nitrato de calcio líquido, Mainstay calcio, sulfato de magnesio, EDTA-Fe 11\%, cosmoquel B y molibdato de amonio.

\section{Muestreos de material vegetal}

Los muestreos se realizaron cada $13 \mathrm{~d}$, seleccionando tres árboles por cada tratamiento (24 por muestreo) por repetición para un total de seis muestreos destructivos realizados a los 33, 46, $59,72,85$ y 98 ddt (días después de trasplante) para todas las variables.

\section{Variables evaluadas}

Se determinó la altura de la planta, número de hojas, área foliar (empleando un medidor LI3100 de LI-COR, Lincoln, NE) y peso seco (estufa a $75^{\circ} \mathrm{C}$ durante $48 \mathrm{~h}$ ) de hojas, tallos y raíces.

\section{Análisis estadístico}

Se realizó análisis de varianza Anova y se empleó la prueba de comparación de medias de Tukey con confiabilidad del 95\%. Los cambios de magnitud de las plantas y variables de crecimiento se determinaron mediante el análisis funcional del modelo logístico como describen Flórez et al. (2006), para ello se usaron los programas Microsoft Excel® y software estadístico Statistic System Analysis (SAS) v. 9.1.3.

Tabla 1. Niveles de nutrientes (mg L-1) calidad reactivo para cada tratamiento (Dussán et al., 2015).

\begin{tabular}{|l|r|r|r|r|r|r|r|c|}
\hline \multicolumn{10}{|c|}{ Tratamiento } \\
\hline Nutriente & $-\mathrm{N}$ & $-\mathrm{P}$ & $-\mathrm{K}$ & $-\mathrm{Mg}$ & - - a & $-\mathrm{B}$ & Completa & Agua destilada \\
\hline $\mathrm{N}$ & 0 & 43 & 43 & 43 & 43 & 43 & 43 & 0 \\
\hline $\mathrm{P}$ & 12 & 0 & 12 & 12 & 12 & 12 & 12 & 0 \\
\hline $\mathrm{K}$ & 40 & 40 & 0 & 40 & 40 & 40 & 40 & 0 \\
\hline $\mathrm{Mg}$ & 7 & 7 & 7 & 0 & 7 & 7 & 7 & 0 \\
\hline $\mathrm{Ca}$ & 7 & 7 & 7 & 7 & 0 & 7 & 7 & 0 \\
\hline $\mathrm{B}$ & 7 & 7 & 7 & 7 & 7 & 0 & 7 & 0 \\
\hline
\end{tabular}




\section{RESULTADOS Y DISCUSIÓN}

\section{Cambios de magnitud de las plantas}

\section{Altura de la planta}

La altura de las plantas sometidas a todos los tratamientos únicamente presentó diferencias significativas $(P \leq 0,05)$ en el último muestreo (a los $98 \mathrm{ddt}$ ). Las plantas con mayor altura correspondieron a las plantas sometidas a deficiencia de magnesio y boro con valores de 59,67 y 58,83 $\mathrm{cm}$, respectivamente (tabla 2 ). Las plantas que recibieron solo agua presentaron una disminución del 35,5\% en tamaño. Las plantas con deficiencias $(-\mathrm{N})$ presentaron una disminución del $12 \%$. Las plantas de los demás tratamientos no presentaron diferencias en su altura por efecto de los tratamientos carenciales. La altura de planta es un cambio de magnitud similar a la tasa de alargamiento de los brotes (TAB) propuesta por Hunt (1990) que es producto del número de brotes por rama y su longitud tomada a diferentes intervalos de tiempo, que se considera una adaptación de la tasa de crecimiento. Esta tasa fue evaluada en guayaba por Nava et al. (2004), quienes encontraron que la $\mathrm{TAB}$ varió ampliamente a lo largo del año desde 0,04 hasta 0,32 $\mathrm{cm} \mathrm{d}^{-1}$ cuya variación estuvo asociada con la humedad del suelo que favorece la división y el alar- gamiento celular, proceso que también depende de la acumulación de reservas por la planta en sus diferentes estructuras como lo determinaron Chadha y Pandey (1986). En guayabo, al igual que en otras especies, la composición foliar de nutrientes varía con la edad de la planta (como se observó en las plantas a los 98 ddt, donde se encontraron diferencias significativas en altura); con el estado fenológico del árbol, el número de brotes por planta (en este experimento fue evidente la reducción en la producción y crecimiento de brotes nuevos durante todo el desarrollo del experimento), similar a lo reportado por Salvador et al., (1998; 1999) en plántulas de esta especie. Dependiendo también del tipo de rama, posición de hojas en la rama, de tal manera que los niveles de N, P, K, Zn y Cu en hojas de guayabo disminuyen a medida que aumenta la edad de la hoja, mientras que el Ca, Mg y Mn se incrementan (Singh y Rajput, 1978).

\section{Número de hojas}

A lo largo de la evaluación se observó que el número de hojas varió significativamente por efecto de los tratamientos. A partir del 59 ddt se evidenció en las plantas tratadas con solo agua y $-\mathrm{N}$ los menores valores en número de hojas, 33 y 34 , respectivamente; en comparación con los demás tratamientos, los cuales presentaron valores en-

Tabla 2. Altura de plantas de guayaba, sometidas a deficiencias de N, P, K, Mg, Ca y B, comparadas con fertilización completa o regadas solamente con agua.

\begin{tabular}{|l|c|c|c|c|c|c|}
\hline \multicolumn{7}{|c|}{ Altura de planta (cm) } \\
\hline \multicolumn{1}{|c|}{ Tratam. } & 33 & 46 & 59 & 72 & 85 & 98 \\
\hline Agua & $45,33 \mathrm{a}$ & $31,01 \mathrm{a}$ & $41,83 \mathrm{a}$ & $43,67 \mathrm{a}$ & $47,00 \mathrm{a}$ & $39,67 \mathrm{~b}$ \\
\hline Compl. & $37,33 \mathrm{a}$ & $32,10 \mathrm{a}$ & $33,33 \mathrm{a}$ & $49,33 \mathrm{a}$ & $54,00 \mathrm{a}$ & $56,67 \mathrm{ab}$ \\
\hline (-N) & $38,50 \mathrm{a}$ & $39,33 \mathrm{a}$ & $41,33 \mathrm{a}$ & $45,67 \mathrm{a}$ & $51,00 \mathrm{a}$ & $52,67 \mathrm{ab}$ \\
\hline (-P) & $38,00 \mathrm{a}$ & $41,00 \mathrm{a}$ & $41,50 \mathrm{a}$ & $53,33 \mathrm{a}$ & $51,33 \mathrm{a}$ & $55,33 \mathrm{ab}$ \\
\hline (-K) & $45,17 \mathrm{a}$ & $41,83 \mathrm{a}$ & $42,00 \mathrm{a}$ & $50,33 \mathrm{a}$ & $57,33 \mathrm{a}$ & $53,67 \mathrm{ab}$ \\
\hline (-Mg) & $37,17 \mathrm{a}$ & $50,67 \mathrm{a}$ & $41,00 \mathrm{a}$ & $51,67 \mathrm{a}$ & $60,50 \mathrm{a}$ & $59,67 \mathrm{a}$ \\
\hline (-Ca) & $50,17 \mathrm{a}$ & $37,17 \mathrm{a}$ & $48,33 \mathrm{a}$ & $56,50 \mathrm{a}$ & $60,00 \mathrm{a}$ & $54,33 \mathrm{ab}$ \\
\hline (-B) & $44,50 \mathrm{a}$ & $28,50 \mathrm{a}$ & $40,67 \mathrm{a}$ & $52,50 \mathrm{a}$ & $54,33 \mathrm{a}$ & $58,83 \mathrm{a}$ \\
\hline
\end{tabular}

Promedios con letras distintas, entre columnas por día de muestreo, indican diferencia significativa según la prueba de Tukey $(P \leq 0,05)$. 
tre 45 y 63 hojas por planta (figura 1). La ausencia de nitrógeno limitó el desarrollo de las hojas en cuanto a su número, lo que generó menor tejido foliar para fotosíntesis y para la producción de fotoasimilados, los cuales son empleados para la generación de nuevos tejidos vegetativos en etapas iniciales; mayor producción de masa seca significa mayor productividad (Marschner, 2011). Se puede asegurar que las deficiencias de $\mathrm{N}$ alteran la nueva formación de hojas y su desarrollo, debido posiblemente a las bajas tasas de producción de aminoácidos y proteínas indispensables en la división y elongación de células (Cabezas y Sánchez, 2008). A los 98 ddt las plantas de menor número de hojas correspondió a aquellas tratadas con agua 28 hojas en promedio con diferencias significativas $(P \leq 0,05)$, seguido por las plantas con deficiencia de $\mathrm{Mg}(-\mathrm{Mg})$, siendo estos dos tratamientos los de mayor afectación. Las plantas con mayor número de hojas, 103 en promedio, fueron las de plantas con el tratamiento (-Ca), seguidas por las plantas con el tratamiento con fertilización completa.

Según Havlin et al. (1999) y Kalaji et al. (2014), el nitrógeno, además de tener función estructural en la molécula de clorofila, participa también en la síntesis proteica, la inhibición de esta síntesis reduce el proceso de división celular, y como resultado afecta el crecimiento de la planta y emisión de nuevos órganos vegetativos (Marschner, 2012), resultados similares fueron encontrados por Fonseca de Souza et al. (2012) en plántulas de anón amazónico (Rollinia mucosa L.) y asaí (Viégas et al., 2004).

Por otro lado, a partir del $59 \mathrm{ddt}$, las plantas del tratamiento -B presentaron el mayor número de hojas, sin embargo, no presentaron diferencias significativas con las plantas de $-\mathrm{Mg}$ y $-\mathrm{Ca}$.

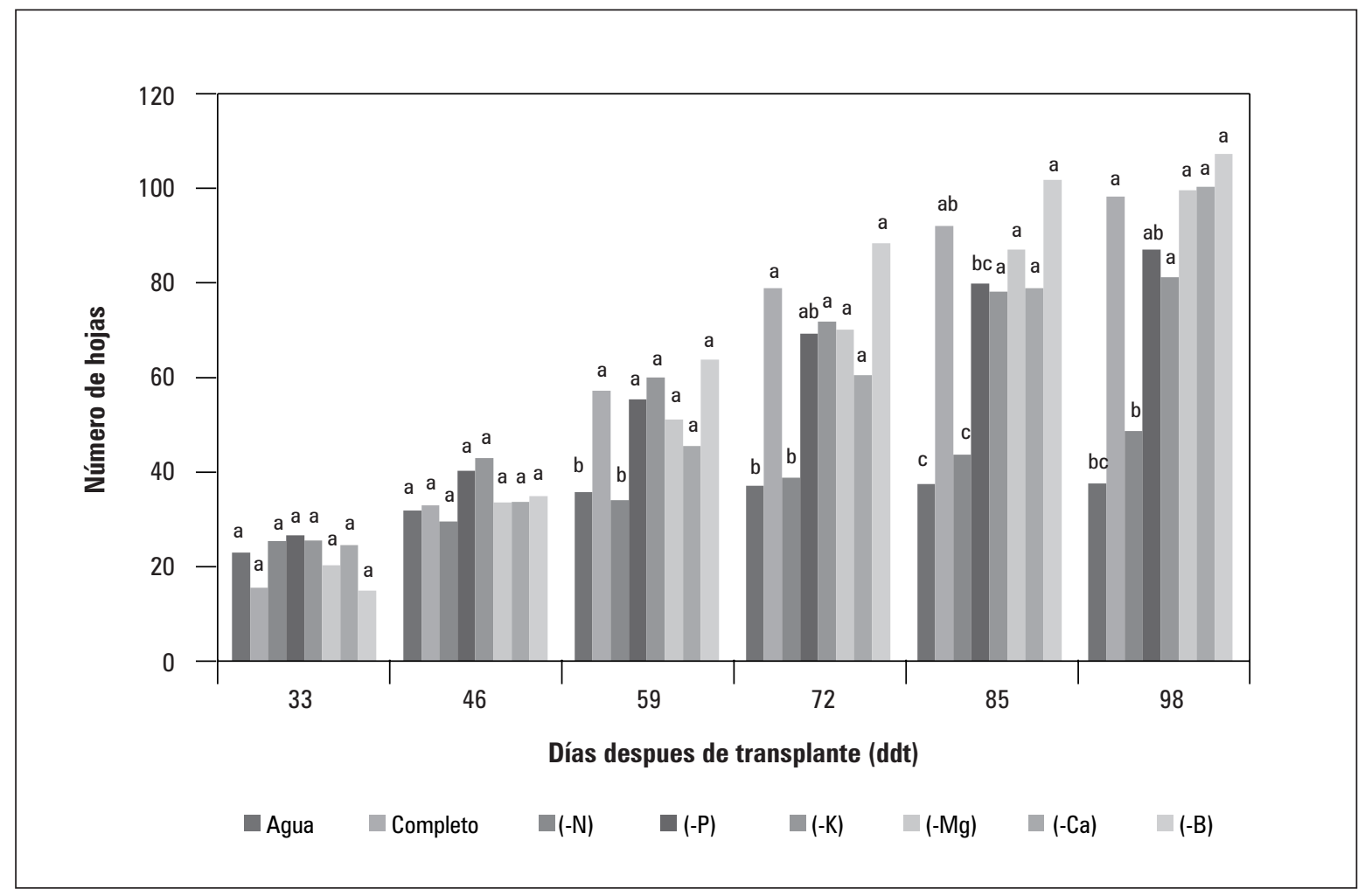

Figura 1. Número de hojas de plantas de guayaba sometidas a deficiencia de $\mathrm{N}, \mathrm{P}, \mathrm{K}, \mathrm{Mg}$, Ca y $\mathrm{B}$, comparadas con fertilización completa o regadas solamente con agua. Promedios con letras distintas, por día de muestreo, indican diferencia significativa según la prueba de Tukey $(P \leq 0,05)$. 
Mendoça et al. (2011) encontraron que plantas de maracuyá deficientes de boro presentaron superbrotación en las regiones apicales debido a la pérdida de dominancia apical, asociado con alteraciones morfológicas y cambio en la diferenciación de tejidos, que a su vez tiene efectos sobre el metabolismo de fenoles, los cuales se acumulan ante una deficiencia del elemento, teniendo efecto no solo en la inhibición de la elongación de la raíz, sino que también induce cambios morfológicos en el crecimiento de la planta (Alarcón, 2001).

\section{Área foliar}

Las plantas sometidas a deficiencia de nitrógeno presentaron un comportamiento casi constante con tendencia a mantener valores bajos en su área foliar, de tal forma que, para el 33 ddt esta fue de 295,0 $\mathrm{cm}^{2}$, sin presentar ninguna variación con respecto a las plantas con fertilización completa; solo a partir del $46 \mathrm{ddt}$, las plantas del tratamiento de $-\mathrm{N}$ comenzaron a presentar reducción de esta variable con 403,5 $\mathrm{cm}^{2}$ con respecto a $443,7 \mathrm{~cm}^{2}$ de las plantas sin carencia de nutrientes (figura 2), lo que representó un 9\% menos ante la omisión de este macronutriente, la reducción aumentó a lo largo del periodo evaluado hasta alcanzar en el 98 ddt, 58,5\%; esto, como resultado de los $547,6 \mathrm{~cm}^{2}$ de área foliar de plantas del tratamiento $-\mathrm{N}$, contra los 1.320,9 $\mathrm{cm}^{2}$ de aquellas con fertilización completa. De igual manera se observó que aquellas plantas del tratamiento solo agua a los $46 \mathrm{ddt}$ presentaron $41,2 \%$ menos de área foliar $\left(246,6 \mathrm{~cm}^{2}\right)$ mientras que a los $98 \mathrm{ddt}$ se produjo incremento con lo que llegó hasta 63,9\% que correspondió a 476,3 $\mathrm{cm}^{2}$. El comportamiento de esta variable pudo deberse principalmente a que, en la fase de desarrollo inicial, las plantas invierten más en la producción de biomasa de hojas, posiblemente para incrementar el área foliar y la tasa fotosintética y de esta forma la biomasa total de la planta (Dias et al., 2008), lo cual tiene relación con una disminución en la emisión de hojas nuevas y un menor crecimiento de estas.

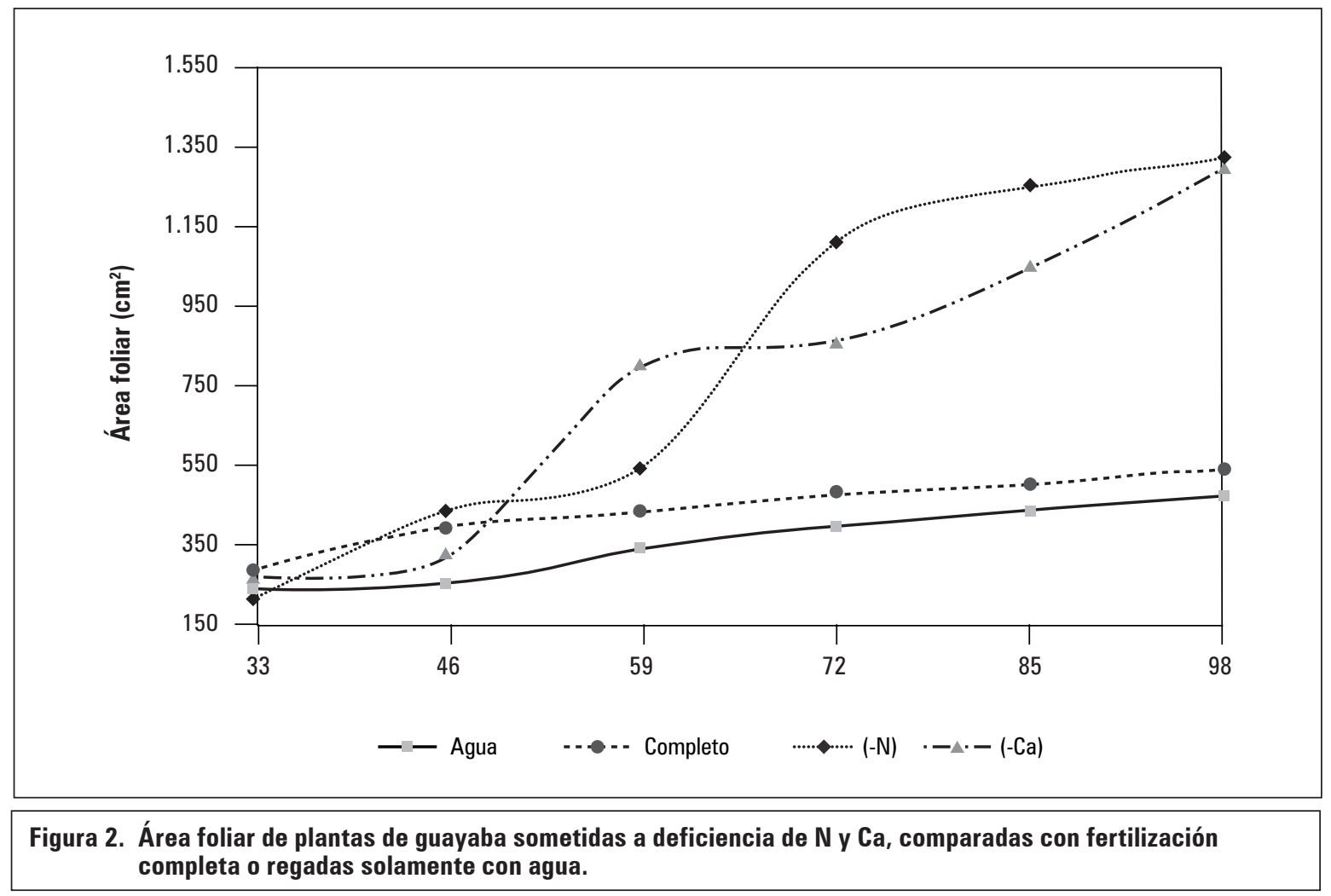

Rev. Colomb. Cienc. Hortic. 
En contraste, aquellas plantas a las cuales no se les suministró calcio (-Ca) se destacaron por presentar la menor variación de área foliar con respecto al tratamiento de las plantas con fertilización completa, de tal forma que desde el 33 ddt hasta el 46 ddt mostraron valores bajos de área foliar partiendo de $277,0 \mathrm{~cm}^{2}$ hasta alcanzar los $333,6 \mathrm{~cm}^{2}$, lo que correspondió a una disminución del 24,1\%; posterior a ello, desde el 46 al 59 ddt se observó un incremento para finalmente entre el 72 al 98 ddt presentar aumento de la variable en todos los puntos muestrales, hasta llegar a los 1.297,9 $\mathrm{cm}^{2}$ al final del experimento, en contraste con los $1320,9 \mathrm{~cm}^{2}$ alcanzados en este mismo punto muestral por las plantas con fertilización completa, lo cual correspondió a tan solo $1,7 \%$ de reducción en este variable; la sintomatología de las plantas deficientes en calcio incluye la deformación de las hojas, reducción de tamaño, disminución en el número (Marschner, 2012).

Por otra parte, las plantas de los demás tratamientos presentaron menor reducción del área foliar al comparárseles con la presentada por las plantas de los tratamientos de solo agua $\mathrm{y}-\mathrm{N}$ en comparación con los valores presentados por las pertenecientes al tratamiento de fertilización completa; -K (8\%), -P (20\%), -Mg (16\%) y -B $(5,4 \%)$. Este comportamiento pudo deberse al hecho de que estos elementos hacen parte de procesos y rutas metabólicas, en las cuales, la carencia de los mismos presenta efecto negativo sobre la generación y expansión del tejido foliar; pero no en la misma magnitud que la deficiencia de nitrógeno.

\section{Distribución de la masa seca por las plantas}

En cuanto a la distribución de masa seca en las plantas, a los 98 ddt se observó que, aquellas plantas del tratamiento de $-\mathrm{N}$ tuvieron menor acumulación de materia seca en tallo y hojas con 22,34 y $12,58 \%$, respectivamente, el restante (65\%) correspondió a la acumulación por la raíz. De igual manera el tratamiento -P, las plantas acumularon un $50 \%$ en raíz, $30 \%$ en hojas y $20 \%$ en tallo. Esta respuesta está posiblemente relacionada con bajas cantidades de fosfato inorgánico en el citosol que pueden disminuir la síntesis de ATP, afectación de la síntesis de la rubisco, generando disminución de las tasas de carboxilación y menor producción de carbohidratos en la hoja, y una mayor acumulación de materia seca en las raíces para favorecer la toma de P y así mitigar el efecto carencial de este nutriente (Wang et al., 2002; Calderón y Moreno, 2009).

En plantas deficientes en $\mathrm{N}$ se produce una reducción de las tasas fotosintéticas como una consecuencia directa de la acumulación de azúcares, que supera la cantidad necesaria para lograr el equilibrio metabólico entre hojas y órganos demanda (Sinclair, 1990; Guo et al., 2007). De forma general, las deficiencias de $\mathrm{N}$ y $\mathrm{P}$ inducen una acumulación de carbohidratos en las hojas, grandes cantidades de $\mathrm{C}$ distribuido a las raíces y un incremento en la relación alométrica raíz/parte aérea (Gutiérrez-Colomer et al., 2006). Estas mismas deficiencias alteran varios eventos fisiológicos, especialmente la fotosíntesis, el metabolismo de azúcares y la asignación de fotoasimilados entre fuentes y demandas (Hermans et al., 2006; Calderón y Moreno, 2009). La menor acumulación de masa seca en la raíz se presentó en el tratamiento -Ca, esto se debe posiblemente a que el Ca estimula la división celular en los ápices meristemáticos, facilitando la división celular, respuesta asociada con su papel en la síntesis de auxinas (Ridge, 1993; Marschner, 2012).

Las plantas a las que se les aplicó solo agua presentaron una distribución en tallo $21,12 \%$, hojas $15,14 \%$ y en raíz $63,73 \%$. Las plantas con fertilización completa fueron las que registraron una relación parte aérea: raíz más cercana, pues $25,13 \%$ fue distribuida hacia los tallos, $31,22 \%$ hacia las hojas y 43,64\% hacia las raíces. Estos resultados concuerdan con lo reportado por Facincani et al. (2007), quienes encontraron en plántulas de guayaba cvs. Paluma y Século XXI, una distribución de materia seca de hojas (56\%), tallo $(29 \%)$ y raíces $(15 \%)$, con lo que se podría 
constatar que la mayor acumulación de materia seca en plántulas de guayaba en fase inicial bajo condiciones óptimas de fertilización; es decir, sin deficiencia de elementos esenciales para su crecimiento y desarrollo, se realiza en tejido foliar (figura 3). Uno de los mecanismos que utilizan las plantas para ajustar su metabolismo a la deficiencia de recursos exógenos es la modificación de los patrones de distribución de biomasa, en especial, almacenando reservas en los órganos requeridos para buscar y solventar los recursos limitantes (Marschner et al., 1996; Yeh et al., 2000).

\section{Masa seca total acumulada por la planta}

La masa seca total acumulada por la plantas no presentó diferencias en respuesta a los tratamientos evaluados hasta los $59 \mathrm{ddt}$. En el periodo comprendido entre el 59 y $72 \mathrm{ddt}$, las plantas a las cuales solo se les aplicó agua y aquellas sometidas a deficiencia de nitrógeno (-N), presentaron una reducción de su masa seca acumulada del 25,8 y $16 \%$ respectivamente, mientras a los 98 ddt la reducción fue del 35 y 28,9\% para estos mismos tratamientos (figura 4)
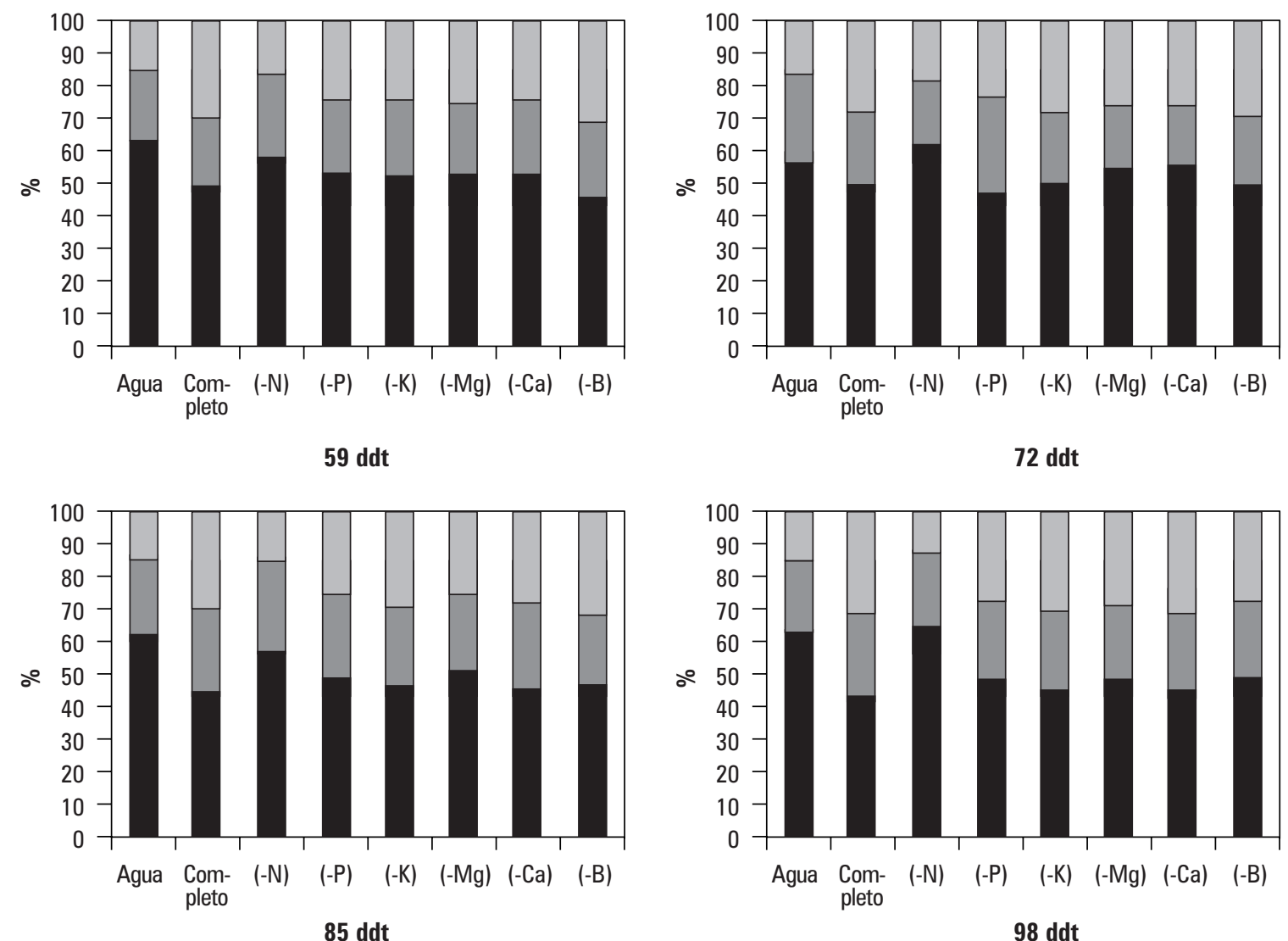

\footnotetext{
\[ \text { Hojas } \square \text { Tallo } \]
Figura 3. Distribución de masa seca, hojas, tallo y raíz en plantas de guayaba sometidas a deficiencia de $\mathbf{N}, \mathbf{P}, \mathrm{K}$,
Mg, Ca y $\mathrm{B}$, comparadas con fertilización completa o regadas solamente con agua. ddt = días después de transplante.
} 


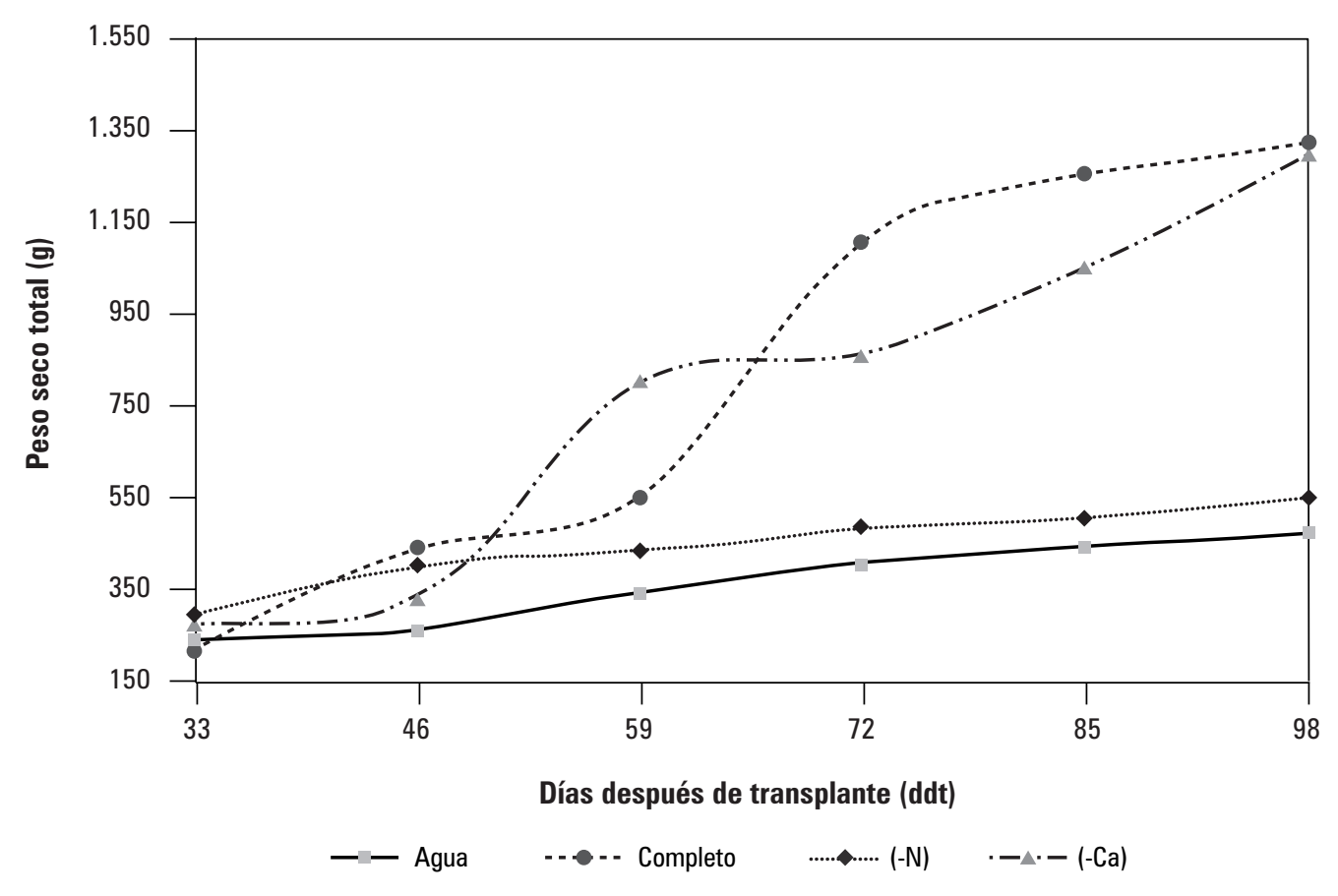

Figura 4. Promedios de biomasa seca total acumulada en plantas de guayaba sometidas a deficiencia de $\mathrm{N}$ y $\mathrm{Ca}$, comparadas con fertilización completa o regadas solamente con agua.

Guo et al. (2007) y Lizarazo et al. (2013b) describen que cuando se presenta deficiencia en $\mathrm{N}$ en las plantas, estas pueden acumular azúcares y almidón en hojas y luego comenzar el transporte de sacarosa hacia las raíces, pudiéndose presentar disminución en la producción de fotoasimilados por efecto de la acumulación de carbohidratos de reserva en la hoja, además de un posible efecto negativo sobre la relación $\mathrm{C} / \mathrm{N}$, lo que generaría disminución del $\mathrm{N}$ utilizado para la regeneración de la rubisco, y como respuesta se generaría afectación en el proceso fotosintético; además de presentarse carencia de bases nitrogenadas las que impiden la activación de la división celular y acción de los genes (Hermans et al., 2006; Guo et al., 2007). Resultados similares fueron encontrados en plántulas de curuba (Passiflora tripartita) (Cabezas y Sánchez, 2008).

Así mismo, durante el periodo comprendido entre 33 a 59 ddt, las plantas sometidas a deficiencia de boro fueron las que registraron los valores mayores, resultado que contradice lo encontrado por Lizarazo et al. (2013b), quienes encontraron que ante la deficiencia de boro las plantas de curuba presentaron menor acumulación de masa seca. Para el 72 ddt, no se evidenció reducción en la acumulación de masa seca total en la planta, pues presentaron 37,0 g/planta, en comparación de las plantas con fertilización completa (36,8 g/planta). De igual manera, a los $98 \mathrm{ddt}$, las plantas con carencia de boro presentaron una reducción del $1,6 \%$ con respecto a aquellas con fertilización completa que alcanzaron valores de 51,1 g/planta. Salvador et al. (2003), en un estudio realizado en plántulas de guayaba variedad nativa no especificada, corroboraron que el boro no se presenta como un nutriente que limite la producción en fase de vivero, debido a que no se presentó diferencias significativas en la acumulación de materia seca de la planta en función de la aplicación de seis diferentes dosis $(0 ; 0,125 ; 0,25 ; 0,5 ; 1,0 ; 1,5$ y 3 $\left.\mathrm{mg} \mathrm{L}^{-1}\right)$ de este nutriente. 
Para las plantas de los tratamientos con deficiencia de los nutrientes $\mathrm{P}, \mathrm{K}, \mathrm{Mg}$ y Ca se presentó disminución en la acumulación de masa seca en comparación con las plantas del tratamiento de fertilización completa, sin embargo, la reducción no fue significativa comparada con la respuesta obtenida con el tratamiento (-N).

\section{CONCLUSIONES}

El nutriente más limitante para el crecimiento y la acumulación de masa seca por las plantas de guayaba en fase de vivero (10 meses de edad) fue el $\mathrm{N}$, ya que su carencia afectó negativamente el número de hojas, el área foliar y la acumulación de masa seca por las plantas.

El efecto de la carencia total de nutrientes en plantas de guayaba en fase de vivero tuvo como efecto el retraso de crecimiento principalmente del número de hojas y del área foliar, pero no condujo a la muerte de estas.

En respuesta a los elementos carenciales, las plantas de guayaba respondieron con la redistribución diferencial de la masa seca hacia diferentes órganos, beneficiando la acumulación de la masa seca en raíces, seguida por las hojas y finalmente por el tallo.

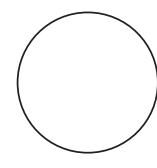

Alarcón, A. 2001. El boro como nutriente esencial. Ediciones de horticultura. En: Horticom, http:// www.horticom.com/pd/imagenes/51/155/51155. pdf; consulta: febrero de 2015.

Bruulsema, T.W., P.E. Fixen y G.D. Sulewski (eds.). 2012. Plant nutrition manual: A manual for improving the management of plant nutrition, metric version. International Plant Nutrition Institute (IPNI), Norcross, GA.

Benincasa, M.M.M. 2003. Análise de crescimento de plantas (Nociones básicas). $2^{\circ}$ ed. FUNEP, Jaboticabal, Brasil.

Cabezas, M. y C. Sánchez. 2008. Efecto de las deficiencias nutricionales en la distribución de la materia seca en plantas de vivero de curuba (Passiflora mollissima Bailey). Agron. Colomb. 26, 197-204.

Calderón, A. y E. Moreno. 2009. Producción de frutos de guayaba (Psidium guajava L.) variedad Taiwan 1, utilizando diferentes programas de fertilización N-P-K. Trabajo de grado. Facultad de Ciencias Agropecuarias, Universidad de El Salvador, San Salvador.

Campbell, C.R. 2000. Reference sufficiency ranges for plant analysis in the southern region of the United States. North Carolina Department of Agriculture and Consumer Services, Raleigh, NC.

\section{REFERENCIAS BIBLIOGRÁFICAS}

Chadha, L.K y M.R. Pandey. 1986. Psidium guajava L. pp. 287-293. En: Halevy, A.A. (ed.). Handbook of flowering. Vol. 5. CRC Press, New Delhi, India.

Dias, L., R. Demello, D. Rozaney N. Freitas. 2008. Acúmulo de massa seca e marcha de absorção de nutrientes em mudas de goiabeira (Pedro sato). Bragantia 67(3), 577-587. Doi: 10.1590/S0006-87052008000300004

Dussán C., S.L., D.A. Villegas y D. Miranda L. 2015. Análisis de crecimiento de árboles de guayaba ( $P$ sidium guajava L.) var. ICA Palmira II sometidos a deficiencia de N, P, K, Mg, Ca y B en fase de vivero. Rev. Colomb. Cienc. Hortic. 9(2), 209-221. Doi: 10.17584/rcch.2015v9i2.4176

Facincani, C., R. Demello, L. Brachirolli y D. Rozane. 2007. Curva de crescimento e marcha de absorção de macronutrientes em mudas de goiabeira. R. Bras. Ci. Solo 31, 1429-1437. Doi: 10.1590/S0100-06832007000600020

FAO. 2013. FAO statistics. FAO, Roma.

Fischer, G., L.M. Melgarejo y D. Miranda. 2012. Guayaba. pp. 526-549. En: Fischer, G. (ed). Manual para el cultivo de frutales en el trópico. Produmedios, Bogotá.

Flórez, V., D. Miranda, B. Chaves, L. Chaparro, C. Cárdenas y A. Farías. 2006. Parámetros considerados en el análisis del crecimiento en rosa y clavel en 
los sistemas de cultivo en suelo y en sustrato. pp. 43-51. En: Flórez, V., A. Fernández, D. Miranda, B. Chaves y M. Guzmán (eds.). Avances sobre fertirriego en la floricultura colombiana. Unibiblos, Bogotá.

Fonseca de Souza, M., M. Queiroz, M. De Oliveira y R. Inácio. 2012. Omissão de macronutrientes em mudas de biribazeiro (Rollinia mucosa [Jacq.] Baill) cultivadas em solução nutritiva. Agron. Colomb. $30(1), 41-45$.

Guo, S., Y. Zhou, X. Gao, Y. Li y Q. Shen. 2007. New insights into the nitrogen form effect on photosynthesis and photorespiration. Pedosphere 17(5), 601-610. Doi: 10.1016/S1002-0160(07)60071-X

Gutiérrez-Colomer, R.P., M.M. González-Real y A. Bai1le. 2006. Dry matter production and partitioning in rose (Rosa hybrida) flower shoots. Sci. Hortic. 107, 284-291. Doi: 10.1016/j.scienta.2005.08.003

Havlin, J.L, J.D. Beaton, S.L. Tisdale y W.R. Nelson. 1999. Soil fertility and fertilizers - An introduction to nutrient management. $6^{\text {th }} \mathrm{ed}$. Prentice Hall, New Jersey, NJ.

Hermans, C., J. Hammond, P. White y N. Verbruggen. 2006. How do plants respond to nutrient shortage by biomass allocation?. Trends Plant Sci. 11(12), 611-617. Doi: 10.1016/j.tplants.2006.10.007

Hoagland, D.R. y D.I. Arnon. 1950. The water-culture method for growing plants without soil. College of Agriculture, Agricultural Experiment Station, University of California, Berkeley, CA.61

Hunt, R. 1990. Basic growth analysis. Unwin Hyman, London.

IIFT (Instituto de Investigaciones de Fruticultura Tropical). 2011. Instructivo técnico para el cultivo de la guayaba. La Habana.

Kalaji, H., A. Oukarroum, V. Alexandrov, M. Kouzmanova, M.Brestic, M. Ziycak, IA. Samborska, MD. Cetner, SI. Allakhverdiev y V. Goltsev. 2014. Identification of nutrient deficiency in maize and tomato plants by in vivo chlorophyll a fluorescence measurements. Plant Physiol. Biochem. 81, 16-25. Doi: 10.1016/j.plaphy.2014.03.029

Lizarazo, M., C. Hernandez, G. Fischer y M. Gómez. 2013a. Response of the banana passion fruit (Passiflora tripartita var. mollissima) to different levels of nitrogen, potassium and magnesium. Agron. Colomb. 31, 44-54.

Lizarazo, M., C. Hernández, G. Fischer y M. Gómez. 2013b. Biomasa, parámetros foliares y sintomatología en respuesta a diferentes niveles de manganeso, zinc y boro en curuba (Passiflora tripartita var. mollissima). Rev. Colomb. Cienc. Hort. 7, 31-45 . Doi: 10.17584/rcch.2013v7i1.2033

Malavolta, E. 2006. Manual de nutrição de plantas. Ceres, São Paulo, Brasil.

Marschner, H.E., A. Kirkby e I. Cakmak. 1996. Effect of mineral nutricional status on shoot-root partitioning of photoassimilates and cycling of mineral nutrients. J. Exp. Bot. 47, 1255-1263 . Doi: 10.1093/ jxb/47.Special_Issue.1255

Marschner, P. (ed.). 2012. Marschner's mineral nutrition of higher plants. $3^{\text {rd }}$ ed. Academic Press, London.

Mendoça, M., P. Monnerat, A. Cordeiro y M. Da Silva. 2011. Sintomas visuais de deficiência de macronutrientes e boro em maracujazeiro-doce. Rev. Bras. Frutic. 33(4), 1329-1349. Doi: 10.1590/S010029452011000400034

MADR. 2007. Acuerdo nacional de competitividad cadena de la guayaba. Ministerio de Agricultura y Desarrollo Rural, Bogotá.

MINAG. 2011. Instructivo técnico del cultivo de la guayaba var. Enana Roja Cubana. Ministerio de la Agricultura. Ed. PALMA-PNUD, La Habana.

Munson, R.D. y W.L. Nelson. 1986. Principles and practices in plant analysis. pp. 223-248. En: Walsh L.M. y J.D. Beaton (eds.). Soil testing and plant analysis. $6^{\text {th }}$ Ed. SSSAJ, Madison, WI.

Natale, W., E. Coutinho, A. Boaretto y F. Pereira. 1996. Goiabeira: Calagem e adubação. FUNEP, Jaboticabal, Brasil.

Nava, A.D., V.A. González, P. Sánchez, C.B. Valdivia, M. Livera y T. Brito. 2004. Crecimiento y fenología del guayabo (Psidium guajava L.) cv. 'Media China' en Iguala. Rev. Fitotec. Mex. 27(4), 349-358.

Norero, A.L. y M.A. Pilatti. 2002. Enfoque de sistemas y modelos agronómicos. Necesidades, métodos y objetos de estudio. Universidad Nacional del Litoral, Santa Fe, Argentina.

Prakash.D., P. Narayana-Swamy y S. Sondur. 2002. Analysis of molecular diversity in guava using RAPD markers. Hort. Sci. Biotech. 77, 287-293.

Ridge, I. 1993. Plant physiology. Hodder \& Stoughton and the Open University, Sevenoaks, UK.

Salvador, J., A. Moreira, E. Malavolta y C. Cabral. 2003. Influência do boro e do manganês no crescimento e na composição mineral de mudas de goiabeira. Ciênc. Agrotec. 27(2), 325-331. Doi: 10.1590/S141370542003000200011

Salvador, J., A. Moreira y T. Muraoka. 1998. Deficiência nutricional em mudas de goiabeira decorrente da 
omissão simultânea de dois macronutrientes. Pesq. Agropec. Bras. 10(33), 1623-1631.

Schachtman, D.P. y R. Shin. 2007. Nutrient sensing and signaling: NPKS. Annu. Rev. Plant Biol. 58, 47-69. Doi: 10.1146/annurev.arplant.58.032806.103750

Sinclair, T.R. 1990. Nitrogen influence on the physiology of crops yield. pp. 41-55. En: Rabbinge, R., J. Goudriaan, H. van Keulen, F.W.T. Penning de Vries y H.H. van Laar (eds.). Theoretical production ecology: reflections and prospects. Pudoc, Wageningen, The Netherlands.

Singh, N. y C. Rajput. 1978. Effect of leaf age and position and fruiting status on guava leaf mineral composition. J. Hort. Sci. 53, 74-74. Doi: 10.1080/00221589.1978.11514797
Viégas, I., M. Thomaz, J. Silva, E. Conceição y A. Naiff. M. 2004. Efeitos da omissão de macronutrientes e Boro no crescimento, nos sintomas de deficiência nutricionais e na composição mineral de plantas de camucamuzeiro. Rev. Bras. Frutic. 26(2), 315 319 . Doi: 10.1590/S0100-29452004000200032

Wang, Y.-H., D.F. Garvin y L. Kochian. 2002. Rapid induction of regulatory and transporter genes in response to phosphorus, potassium, and iron deficiencies in tomato roots. Evidence for cross talk and root/rhizosphere-mediated signals. Plant Physiol. 130, 1361-1370. Doi: 10.1104/pp.008854

Yeh, D.M., L. Lin y C.J. Wright. 2000. Effects of mineral nutrient deficiencies on leaf development, visual symptoms and shoot/root ratio of Spathiphyllum. Sci. Hortic. 86, 223-233. Doi: 10.1016/S03044238(00)00152-7 